CERN-PPE/95-63

30 March 1995

\title{
REVIEW OF THE DEVELOPMENT OF CESIUM IODIDE PHOTOCATHODES FOR APPLICATION TO LARGE RICH DETECTORS
}

\author{
J. Almeida ${ }^{1)}$, A. Amadon²), P. Besson ${ }^{2)}$, P. Bourgeois' ${ }^{2)}$, A. Braem ${ }^{3)}$, \\ A. Breskin ${ }^{4}$, A. Buzulutskov'), R. Chechik ${ }^{4)}$, C. Coluzza1), A. Di Mauro ${ }^{5)}$, J. Friese ${ }^{6}$, \\ J. Homolka6), A. Ljubicic Jr. ${ }^{7)}$, G. Margaritondo'), Ph. Miné8), E. Nappi ${ }^{5}$, \\ T. dell'Orto ${ }^{1}$, G. Paic ${ }^{9)}$, F. Piuz ${ }^{3, *)}$, F. Posa ${ }^{5)}$, J.C. Santiard ${ }^{3)}$, S. Sgobba ${ }^{3)}$, \\ G. Vasileiadis ${ }^{8)}$ and T.D. Williams ${ }^{3)}$
}

CERN-RD 26 Collaboration

\begin{abstract}
CsI photocathodes were studied in order to evaluate their potential use as large photo converters in RICH detectors for the PID system of ALICE at LHC in heavy-ion collider mode. It has been demonstrated that a quantum efficiency close to the reference value obtained on small samples can be obtained on CsI layers evaporated on large pad electrodes operated in a MWPC at atmospheric pressure. We present a survey of the results obtained in the laboratory on small samples irradiated with UV-monochromatic beams and with large area RICH detectors of proximity-focusing geometry in a $3 \mathrm{GeV} / c$ pion beam.
\end{abstract}

Presented at the Wire Chamber Conference, Vienna, Feb. 1995

to be published in Nuclear Instruments and Methods

\footnotetext{
*) Corresponding author.

1) Ecole Polytechnique Fédérale, Lausanne, Switzerland.

2) CEN Saclay, DAPNIA, Gif/Yvette, France.

3) CERN, Geneva, Switzerland.

4) The Weizmann Institute, Rehovot, Israel.

5) INFN and Politecnico, Bari, Italy.

6) Munich Technische Universität, Garching, Germany.

7) The Ruder Boskovic Institute, Zagreb, Croatia.

8) Ecole Polytechnique, LPNHE, IN2P3-CNRS, Palaiseau, France.

9) SUBATECH, Nantes, France.
} 


\section{INTRODUCTION}

The aim of the RD-26 collaboration is to develop large-area systems for particle identification, based on fast CsI-RICH modules. They have to cope with very high event multiplicities met in heavy ion experiments - for example, ALICE at the LHC operated in the lead ion collider mode [1]. This article reviews the principal results obtained during the past two years covering two main aspects. Firstly, the quantum efficiency (QE) of small CsI samples, studied by measuring the photocurrent or by counting the photoelectrons emitted under exposure to VUV-monochromatic light in vacuum and under gas. Secondly, the performance of large area CsI photocathodes (PC) in a RICH detector. The QE, as well as the detector performance, were studied with photons emitted from a Cherenkov radiator under relativistic particle irradiation.

\section{BASIC FEATURES OF CSI LAYERS}

Layer processing. Systematic studies of CsI layers deposited on metallic substrates, independently performed by several RD-26 institutions, have confirmed [2] that a reproducible QE performance can be achieved in a vacuum when the deposits are produced according to the following procedure. The CsI is evaporated by Joule effect within ranges of substrate pressure, rate and temperature of a few $10^{-7}$ to $10^{-6}$ Torr, 2 to $10 \mathrm{~nm} / \mathrm{sec}$ and 50 to $60^{\circ} \mathrm{C}$, respectively. The substrates are well polished and outgassed at $60^{\circ} \mathrm{C}$ under vacuum before the evaporation. In order to outgas the CsI load, the crucible is steadily heated up to the evaporation temperature for a few minutes. A shutter stops the first emission of CsI vapor and is removed after this period of time. A key element in achieving an optimum QE is to keep the layer under vacuum at $50-60^{\circ} \mathrm{C}$ for several hours after evaporation (Fig. 3 in Ref. [3]). No clear influence of the initial CsI conditioning, powder or crystal, was put into evidence. The dependence of the QE versus wavelength obtained in vacuum (Fig. 3 in Ref. [2]) is shown in Fig. 2 and is taken as a reference QE curve, since it results from a large set of measurements performed under well controlled procedures. Higher QE values may be achieved using a layer processing differing from the one described above - for example, a $20 \%$ increase was obtained on a layer deposited with an electron gun (Figs. 4-10 in Ref. [4]). It has been noticed that the QE is first affected at large wavelengths when processing is of lower quality.

CsI quantum efficiency under gas. The photoelectric yield of CsI layers exposed to a gas has recently been found to depend on the electron transport property of the gas [5]. Beyond the intrinsic QE of CsI, the gas composition and the electrical field strength at the layer surface characterise the photoelectric yield. Compared to measurements in vacuum, the $\mathrm{QE}$ is found to be very little affected in hydrocarbon-based mixtures down to low $\mathrm{E} / \mathrm{p}$ values of $2 \mathrm{~V} / \mathrm{cm}$.Torr. A significant drop in the QE is observed in noble gas-based mixtures - i.e., by a factor of two to three in the case of helium, the vacuum value being restored at $\mathrm{E} / \mathrm{p}>5-8 \mathrm{~V} / \mathrm{cm} \cdot$ Torr. Such a difference in photoemission (at low E/p) between various gases is explained by the different probability for the photoelectron elastic backscattering [6]. That effect influences the choice of gas mixture when operating a CsI photocathode in a wire chamber (MWPC), where the E/p value, at atmospheric pressure, is inherently low at the cathode surface $(<2-4 \mathrm{~V} / \mathrm{cm} \cdot$ Torr $)$.

Very high $\mathrm{E} / \mathrm{p}$ values can be achieved by evaporating the CsI on the thin anode wires of a MWPC. Operating under vacuum, the $\mathrm{QE}$ is found to be enhanced by a large factor, reaching 25 at $\mathrm{E}=5 \times 10^{5} \mathrm{~V} / \mathrm{cm}[7]$. 


\section{FAST RICH AND CsI PHOTOCATHODES}

Detailed descriptions of the CsI-RICH detector and its operation are to be found in Ref. [8]. The CsI layer is deposited on the cathode of a MWPC segmented into pads of size $8 \times 8 \mathrm{~mm}^{2}$. The MWPC, detecting the single electrons emitted from the CsI layer, has a anodecathodes distance of $2 \mathrm{~mm}$ and sense wires of $20 \mu \mathrm{m}$ in diameter, spaced by $4 \mathrm{~mm}$. The second cathode, made of wires or a mesh, defines the active volume of the MWPC. The proximity gap extends up to the radiator to provide the distance necessary for achieving sizeable Cherenkov ring radii. The entire volume is flushed with a UV transparent gas, typically methane. A third wire electrode, positively polarized, is located close to the radiator in order to collect the electrons created by the particles traversing the wide proximity gap. The radiator consists of the liquid $\mathrm{C}_{6} \mathrm{~F}_{14}$ of low chromaticity, contained with a $3 \mathrm{~mm}$-thick quartz window.

\subsection{Influence of the substrate}

In a first series of measurements [8], the CsI evaporation was done on standard printed circuit boards, etching the pad from a $15 \mu \mathrm{m}$ layer of copper on G10 and covered with $0.5 \mu \mathrm{m}$ of chemically deposited gold. On 10 PCs of size $30 \times 30$ and $12 \times 30 \mathrm{~cm}^{2}$, the QE was found to be only $30-50 \%$ of the reference value. In order to develop a pad substrate of large area allowing for higher QE deposits, a large-scale study was undertaken on the various aspects of the surface structure, of the chemical composition of the CsI layers and, finally, of the photoemission properties [9]. Morphological information, given by the scanning electron and tunnelling microscopes (SEM and STM), was compared to the 2-D imaging of the primary or secondary electron emission at a similar lateral resolution (micron range). The latter were obtained with laterally resolved electron microscopy for chemical analysis (ESCA) and photoemission electron microscopy (PEEM). Structural information was also obtained from Xray diffraction measurements. The main results are summarized here. While the CsI layers evaporated on 'high quality' substrates are characterized by a structure composed of contiguous microcrystals with dimensions ranging from 0.3 to $3 \mu \mathrm{m}$, the CsI deposited on 'rough' printed circuit boards exhibit a very non-homogeneous texture. Measurements of the secondary emission have confirmed that close to half of the surface did not emit photoelectrons.

Another particular phenomenon was observed - namely that the X-ray diffraction spectra of CsI evaporated on pure $\mathrm{Cu}$ substrates and on gold-covered copper preferentially exhibited peaks of pure $\mathrm{Cs}$ and pure Iodine instead of peaks belonging to CsI. Many observations were made showing evidence for $\mathrm{Cs} / \mathrm{I}$ segregation over a large range of distances. We surmise that during evaporation $\mathrm{Cu}$ promotes the dissociation of the CsI or prevents its formation from the vapour phase.

Following the findings described above, new, highly-polished boards were produced according to the following procedure: i) production of the pad printed board on G10 and copper only, using standard etching technique, ii) alumina paste and chemical polishing of the copper, iii) chemical deposition of a thick homogeneous nickel layer $(15 \mu \mathrm{m})$; a thinner gold layer was also added on some PCs. CsI layers deposited on such substrates were found to be identical in surface quality and photoemission properties to those deposited on metallic samples.

\subsection{QE of CsI deposited on the new substrates}

The method is based on counting the number of photoelectrons per ring that are a function of the QE as expressed by the Frank-Tamm formula [8]. The localization of the single electrons 
is achieved by measuring the spread of the induction of the avalanche on pads using analog electronics. To every single electron a pad cluster is associated, the mean size of which is typically 1.6 to 2.1 pads depending on chamber gain. Depending on the ring radius and the number of photoelectrons, pad overlap occurs due to the large pad area in use $\left(8 \times 8 \mathrm{~mm}^{2}\right)$. In order to extract the QE, the experimental results are compared with a simulated pad pattern generation, taking into account all the detector features: photon losses calculated from measured UV transparencies and reflectivities of the detector components, CsI QE curve, the spread of induction, and an exponential pulse height generator of fixed mean (SEPH). Single electron pad patterns are overlapped and compared to a threshold, providing the necessary information per ring: total pad pulse height (TPPH), number of pads hit/ring, number of pad clusters, cluster size, etc. Two methods are used to disentangle the pad pattern in terms of individual photons: i) a pad cluster finding analysis [8], efficient in the case of a few photons per ring but limited in the case of large cluster size because of an increasing overlap probability, ii) division of the TPPH measured in a fiducial zone by the mean value of SEPH. The SEPH distribution, expected to be of exponential shape, is extracted from a selection of well separated single electron clusters [8]. To achieve this, we measured events with only a few photoelectrons per ring, reducing the thickness of the radiator.

The results. Five new PCs were tested at a $3 \mathrm{GeV} / c$ pion/proton beam varying the radiator thickness, the chamber gain and using methane as the chamber gas. In Fig. 1, we show the measurements obtained with a $30 \times 30 \mathrm{~cm}^{2} \mathrm{PC}$. The mean number of pads, of photoelectrons obtained as the ratio TPPH/SEPH and of pad cluster per ring are compared to the simulation. A larger number of photoelectrons/unit length are found at small radiator thickness, due to an increase in the UV transmission at low wavelengths where the QE is the highest. In Fig. 2, we compare to the laboratory reference QE curve (dotted line) the two extreme QE curves used in the simulation to fit the data of the five PCs: the upper one, used for the data of Fig. 1, scales to $90 \%$ of the reference, the lower one to $70 \%$. The hatched area corresponds to the $\mathrm{QE}$ measurements obtained with the old substrates (30 to $50 \%$ of the reference QE).

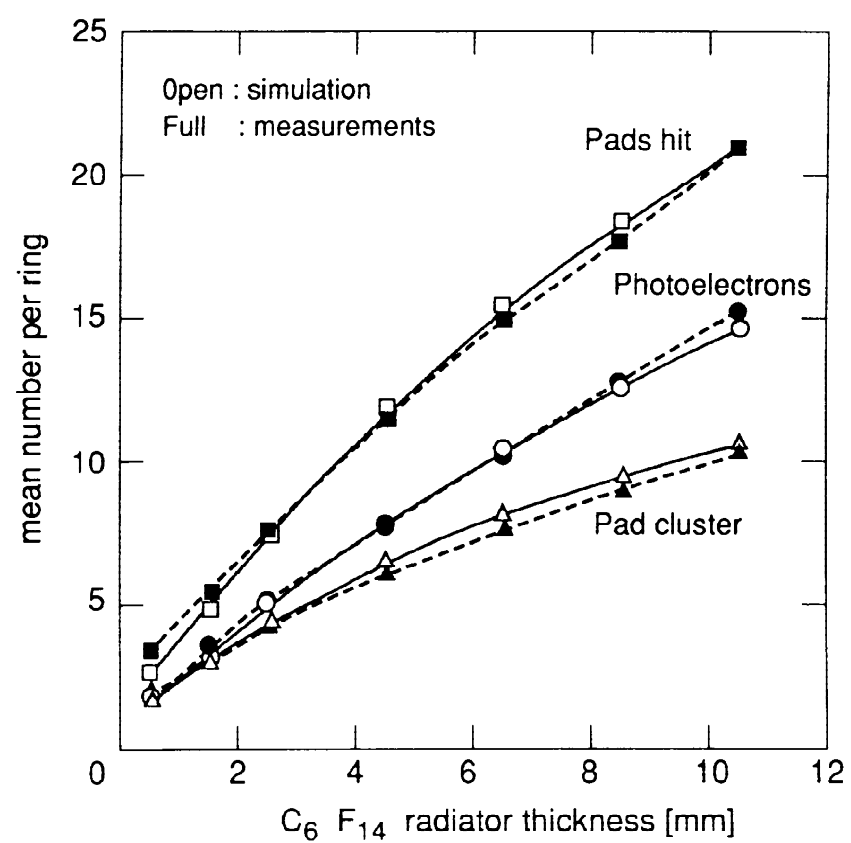

Fig. 1 Results of the CsI-fast RICH using the G10/Cu/Ni/Au pad electrode with $3 \mathrm{GeV} / c$ pions and ring radius of $98 \mathrm{~mm}$ as function of the radiator thickness. 


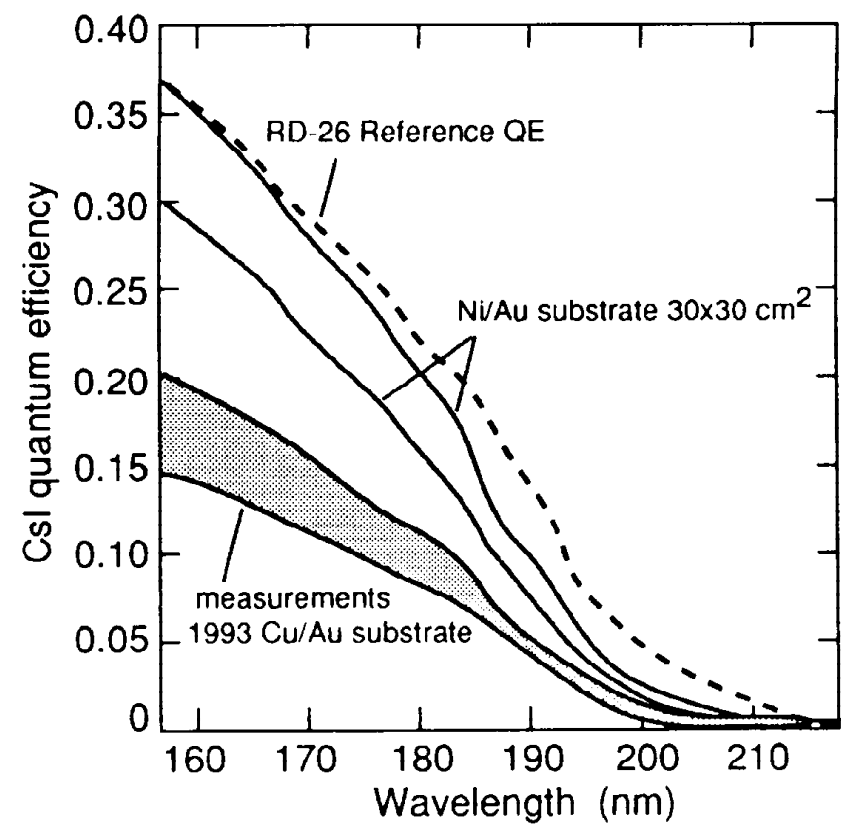

Fig. 2 The CsI QE reference curve (dotted line) compared to QE curves used to fit experimental data. The two upper curves delimit the QE measured on five CsI PCs using the new Ni/Au substrates. The hatched area corresponds to PCs using the old substrates.

\subsection{Performance of the CsI-RICH detector}

Photonic background in an open geometry MWPC and noble gas-based mixtures. An illustration of the photonic background, expected from photon feedback or secondary emissions, is found in Fig. 3, showing frequency distributions of the distances from the ring centre to the centres of any hit pad. In a), a background level corresponding to $0.6-0.8$ photoelectrons/track is superimposed on the Cherenkov peak (14 photoelectrons/track) in case of methane. The b)-distribution, obtained without $\mathrm{C}_{6} \mathrm{~F}_{14}$, can be fitted, assuming an emitting source is located in the remaining $3 \mathrm{~mm}$ quartz window and not in the avalanche of the charged particle associated with the ring. Noble gas-based mixtures were tested in order to possibly reduce the flammable gas load in large systems. We also observed a decrease in the photoelectric yield in RICH operation, accompanied in this case by an increase in the photonic background, up to $>2$ photoelectron/track (Table 1). This is in agreement with the measurements made on small samples (Section 2).

Exposure of CSI PC to air and ageing. A PC was processed, mounted on the RICH and avoiding contact with air, and tested at the beam. Subsequently, no change in performance was observed after exposing the PC to air for one hour. This is corroborated by experiments done on small samples where a decrease in QE of about $30 \%$ was measured after one day's exposure to air [2]. With regard to ageing, a large PC was measured three times at the beam over a fivemonth period, keeping a constant photoelectron yield within experimental fluctuations. This PC was maintained under a methane or argon flow. At Saclay, one small PC was kept under methane flow for three months and another for six months. They were continuously irradiated by an $\mathrm{Fe}^{55}$ source, corresponding to a flux of $3 \times 10^{4} \mathrm{ions} / \mathrm{cm}^{2} / \mathrm{sec}$ impinging the CsI cathode. Their QEs were monitored with a UV lamp and remained stable without any clear degradation being observed over that period. Notice that the irradiation fluxes were very low during these tests. 


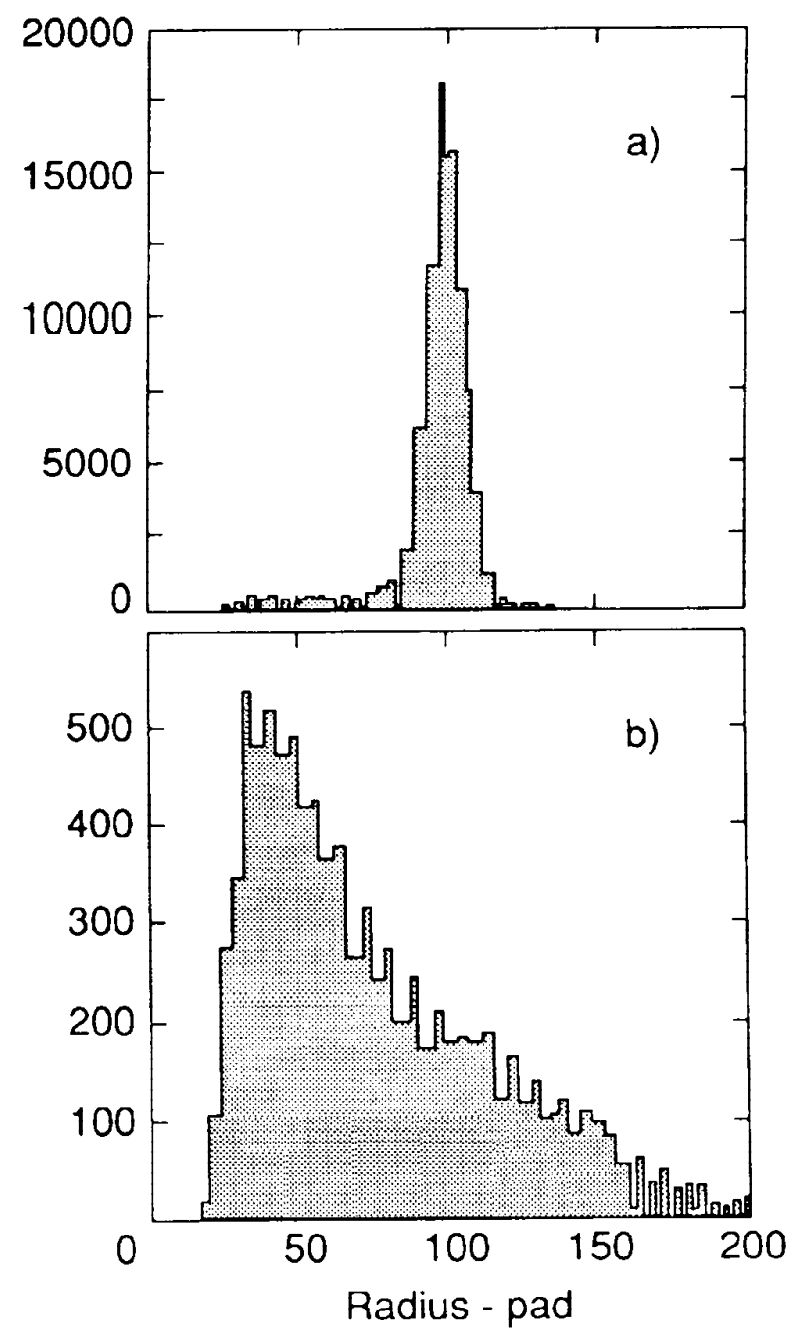

Fig. 3 Radial distributions of pad hits in methane: a) with a $10 \mathrm{~mm} \mathrm{C}_{6} \mathrm{~F}_{14}$ radiator and a $3 \mathrm{~mm}$ quartz plate, illustrating the small background relative to the Cherenkov peak; b) only with the quartz plate, illustrating the shape of the emitting background source (quartz).

Table 1

Effect of the gas mixture on the photonic background and the QE

\begin{tabular}{|c|c|c|}
\hline Gas mixture & $\begin{array}{c}\text { Background/track } \\
\text { (No. photoelectrons) }\end{array}$ & Relative QE \\
\hline $\mathrm{CH}_{4}$ & $0.6-0.8$ & 1.0 \\
$\mathrm{~A} / \mathrm{iC}_{4} \mathrm{H}_{10} 80 / 20$ & 0.8 & $0.75-0.80$ \\
$\mathrm{~A} / \mathrm{CH}_{4} 50 / 50$ & 1.2 & $0.80-0.90$ \\
$\mathrm{~A} / \mathrm{CH}_{4} 80 / 20$ & $>2.0$ & $0.80-0.90$ \\
$\mathrm{He} / \mathrm{iC}_{4} \mathrm{H}_{10} 80 / 20$ & $>3.0$ & $0.50-0.60$ \\
\hline
\end{tabular}

\subsection{Evaluation of the RICH performance at ALICE}

To evaluate the particle identification capability of the present CsI-RICH in a high particle density $\left(50 \mathrm{part} . / \mathrm{m}^{2}\right)$ [10], we proceeded in the following way. We constructed an 'imaginary' $60 \times 60$ pad RICH detector in which we uniformly overlapped the raw events obtained from the actual $30 \times 30 \mathrm{~cm}^{2} \mathrm{RICH}$ prototype until the required density was reached. It is important to 
note that no selection was made on the single events apart from artificially adjusting the proton/pion ratio. To evaluate the performance of the method used to analyse high-density data [11] we first analysed single-ring runs. The $3 \sigma \pi / \mathrm{K}$ separation limit obtained would correspond to $3.3 \mathrm{GeV} / c$ particles. The results of the same procedure applied to the superimposed events are given in Fig. 4. The results shown include only those particles that fell more than $10 \mathrm{~cm}$ away from the detector edges. Note again that the results are very reliable since we did not apply any cuts in the raw data; thus the combined detector and analysis efficiency is $100 \%$ in the fiducial area at the centre of the detector. The RMS is $7.0 \mathrm{mrad}$, which corresponds to a $\pi / \mathrm{K} 3-\sigma$ separation at $2.6 \mathrm{GeV} / c$.

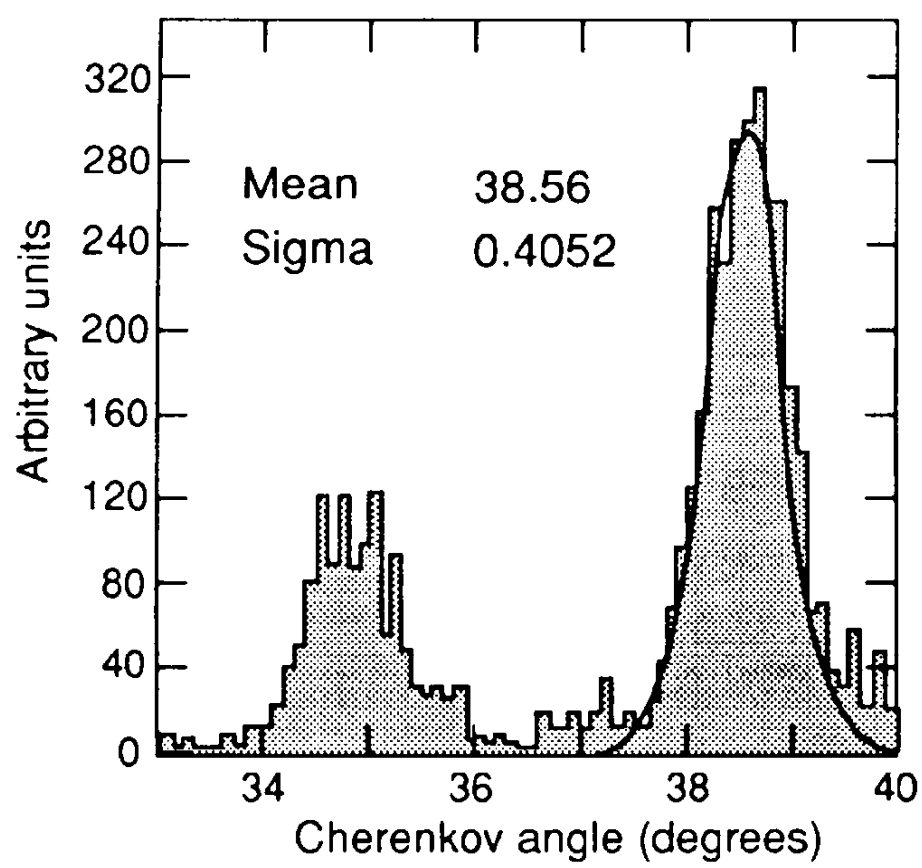

Fig. 4 Results of the analysis of high-density events $\left(50\right.$ part. $\left./ \mathrm{m}^{2}\right)$ using randomly superimposed experimental events obtained with $\pi / \mathrm{p} 70: 30$ of $3 \mathrm{GeV} / c$ momentum.

\section{SUMMARY}

The performance achieved by a fast $\mathrm{RICH}$, using as a photo converter a CsI layer deposited on a novel large area substrate, segmented into pads was:

- 70-90\% of the reference CsI QE in the 160-210 nm range;

- 14 photoelectrons/ring from a $10 \mathrm{~mm}$ thick $\mathrm{C}_{6} \mathrm{~F}_{14}$ radiator with $3 \mathrm{GeV} / \mathrm{c}$ pions (it is expected to reach 16-18/ring with optimized MWPC grid transparencies and final electronics);

- angular resolution for single photoelectron of $8 \mathrm{mrad}$ at $100 \mathrm{~mm}$ ring radius;

- negligible contribution of the photon feedback in an open MWPC geometry in pure methane at a gas gain of up to $1.0 \times 10^{5}$;

- stability of the QE performance after an exposure of the CsI layer to air for up to one hour;

- $\pi / \mathrm{K} 3-\sigma$ separation reaching $2.6 \mathrm{GeV} / c$ from pad patterns, obtained by overlapping test beam events, background included, up to a density of 50 particles $/ \mathrm{m}^{2}$. 
The results obtained so far with the CsI-RICHare very encouraging and demonstrate the feasibility of this technique. More research work in the field of ageing is in progress, in order to better understand the various aspects related to the physics of the CsI photoconverters.

\section{REFERENCES}

[1] Letter of Intent for A Large Ion Collider (ALICE), CERN/LHCC/93-16.

[2] A. Breskin, Secondary emission gaseous detectors. Presented at the 4th International Conference on Advanced Technology and Particle Physics, Como, 1995. Preprint WIS-95/3/Jan.-PH. To be published in Nuclear Physics B Proceedings Supplement.

[3] A. Breskin et al., New ideas in CsI-based photon detectors. Presented at the 1994 IEEE Nucl. Sci. Symposium, Norfolk, Virginia. Preprint WIS-94/47/Oct.-PH. To be published in IEEE Trans. Nucl. Sci.

[4] Proposal for a High-Acceptance Di-Electron Spectrometer, 1994, GSI, Darmstadt. J. Friese, private communication.

[5] A. Breskin et al., Field-dependent photoelectron extraction from CsI in different gases, Preprint WIS-95-4-Feb. PH, this conference.

A. Breskin et al., Nucl. Instrum. Methods A344 (1994) 537.

[6] A. Di Mauro et al., A study of UV-induced photoemission from CsI into gas media, in preparation.

[7] A. Buzulutskov et al., Field enhancement of the photoelectric and secondary electron emission from CsI, to be published in J. of Appl. Phys.

[8] A. Braem et al., Nucl. Instrum. Methods Phys. Res. A343 (1994) 163.

[9] J. Almeida et al., Morphological effects in the quantum yield of CsI, to be published in Nucl. Instrum. Methods Phys. Res. A.

J. Almeida et al., Microanalysis surface and photoemission properties of CsI photocathodes, Preprint WIS-95/14/Mar.-PH, this conference.

[10] J. Almeida et al., Nucl. Instrum. Methods Phys. Res. A348 (1994) 216.

A. Di Mauro et al., Nucl. Instrum. Methods Phys. Res. A343 (1994) 323.

[11] A. Di Mauro et al., Nucl. Instrum. Methods Phys. Res. A343 (1994) 284. 